\title{
Perspectives on endoscopic transsphenoidal surgery
}

\author{
John A. Jane JR., M.D., Joseph Han, M.D., Daniel M. Prevedello, M.D., \\ Jay Jagannathan, M.D., Aaron S. Dumont, M.D., and Edward R. Laws JR., M.D. \\ Departments of Neurological Surgery, Otolaryngology, and Head and Neck Surgery, \\ University of Virginia Health System, Charlottesville, Virginia
}

\begin{abstract}
Sellar tumors are most commonly approached through the transsphenoidal corridor, and tumor resection is most often performed using the operating microscope. More recently the endoscope has been introduced for use either as an adjunct to or in lieu of the microscope. Both the microscopic and endoscopic transsphenoidal approaches to sellar tumors allow safe and effective tumor resection. The authors describe their current endoscopic technique and elucidate the advantages and disadvantages of the pure endoscopic adenomectomy compared with the standard microscopic approach.
\end{abstract}

\section{KEY WORDS • pituitary tumor • transsphenoidal surgery • neuroendoscopy • minimally invasive surgical procedure}

\begin{abstract}
Almost from the outset, the transsphenoidal approach has been minimally invasive. The earliest transsphenoidal surgeries (1907-1909) by Schloffer, ${ }^{19}$ von Eiselsberg, ${ }^{23}$ and Kocher $^{18}$ required external rhinotomy incisions. Endonasal and sublabial approaches were introduced in 1910 by Hirsch $^{12}$ and Halstead, ${ }^{10}$ respectively. These initial procedures were not transseptal and required some degree of middle or inferior turbinectomy, ethmoidectomy, and septectomy. These extensive exposures were required in order to provide both illumination and room for the use of operative instruments.
\end{abstract}

By 1914, however, Cushing ${ }^{4}$ had reported a less traumatic transsphenoidal technique using a sublabial transseptal approach. This was the avenue adopted by Norman Dott, who taught Guiot ${ }^{9}$ - who in turn passed the technique to Hardy. ${ }^{11}$ The primary obstacles to the transsphenoidal approach remained illumination and visualization of the operative field until the late 1960s, when Hardy popularized the use of the operating microscope. Its excellent magnification and illumination capabilities facilitated the effective removal of large and small tumors alike without significant anatomical disruption. The recognition of this potential led to the early 1970 s renaissance of the transsphenoidal approach that has continued until today.

Since the transsphenoidal renaissance, progressive modifications of the microscopic approach have been pursued. At many centers, the sublabial approach has given way to pure endonasal microscopic approaches. The endonasal microscopic transsphenoidal approach has several variations, including the transseptal submucosal technique, the septal pushover, and the direct sphenoidotomy. ${ }^{8,13,24,25}$

Abbreviations used in this paper: $\mathrm{CSF}=$ cerebrospinal fluid; $\mathrm{ENT}=$ ear, nose, and throat.
Essentially, these approaches differ according to the location within the nose where the initial mucosal incision is made and to the width of sellar exposure. The more traditional transseptal submucosal approach is performed using a hemitransfixion incision located just within the nose. The initial incision using the septal pushover is at the junction of the cartilaginous and osseous septum, and the incision using the direct sphenoidotomy is made even farther back, at the rostrum of the sphenoid. The potential benefit of making the incision deeper within the nose is a decrease in both postoperative nasal discomfort and anterior septal complications. The possible drawbacks of the more direct exposures are the more limited exposure, off-midline trajectory, and diminished working room.

More recently, the endoscope has been introduced to transsphenoidal surgery and has gained significant popularity. Endoscope-assisted transsphenoidal operations refer to microscopic procedures in which the endoscope is used as an adjunct to the microscopic removal of a tumor. The manner in which the endoscope is used adjunctively, however, can vary significantly. The endoscope may simply be used to perform an anterior sphenoidotomy prior to inserting the nasal speculum and using the microscope. The endoscope may also be used during the microscopic tumor resection to inspect for areas of tumor residue out of the line of sight of the microscope. Pure endoscopic transsphenoidal surgery refers to the removal of tumors without the use of the operative microscope.

Although endoscope-assisted transsphenoidal approaches had been reported by others, ${ }^{1}$ the pure endoscopic transsphenoidal approach was introduced and popularized in the late 1990s by Jho and Carrau ${ }^{15}$ from the University of Pittsburgh Medical Center. Advances in endoscopic endonasal 
neurosurgical techniques at Pittsburgh continue in the work of Kassam, et al., ${ }^{16,17}$ and Carrau, ${ }^{15}$ who have expanded the scope of complex skull base tumors accessible by the endoscope. In Europe the neurosurgical groups of Cappabianca and de Divitiis ${ }^{2,3}$ and Frank, et al., ${ }^{7}$ have also made important contributions. There are several variations of the endoscopic technique, and over time we have drawn from these to establish the best approach for our patients. We report our current endoscopic technique and detail what we believe are the advantages and limitations of this approach as compared with the microscopic adenomectomy.

\section{Surgical Technique}

Our endoscopic technique has evolved over time as we have acquired instruments and pursued modifications to facilitate exposure and resection. Initially we attempted to recapitulate the microscopic adenomectomy, adopting a mononostril approach with the endoscope holder. We were also more willing to perform partial middle turbinectomies in an effort to create more working room. Our standard approach now is to use a binasal three- or four-hand technique (in lieu of an endoscope holder), with a posterior septectomy preserving the middle turbinates unless the nasal cavity is exceptionally small.

Patients are positioned in a standard semirecumbent position (approximately $20^{\circ}$ back up) with the head placed in a horseshoe headrest as previously described. ${ }^{13}$ Except in repeated transsphenoidal surgery, image guidance is not routinely used and lumbar drains are not routinely placed. ${ }^{5,14}$ Patties soaked in 1:1000 epinephrine are placed between the middle turbinates and the septum prior to preparation and draping. After draping, they are removed, and, using a 25-gauge 1.5-inch or spinal needle, the middle turbinate, posterior portions of the septum and rostrum, as well as the region around the sphenopalatine foramen are injected submucosally with $0.2 \%$ ropivicaine with $1: 200,000$ epinephrine (Fig. 1).

The $0^{\circ}$ endoscope is used to inspect both nasal cavities. The side with the greater working room is chosen for the initial dissection of the posterior septum and sphenoidotomy (if there is a deviated septum, for example, the wider nasal cavity is used). If the sides are equal, our bias is to perform the majority of the dissection in the right naris. Prior to beginning the dissection, the endonasal anatomy is identified, including the middle and inferior turbinates, the choana, and, most important, the superior turbinates and sphenoid ostia. This initial orientation helps to prevent the undesirable breach of the anterior cranial fossa. Indeed, we have found that the novice endoscopist tends to err superiorly. Understanding and recognizing this propensity is an important safeguard. The sphenoid ostium is typically seen in the inferior third portion of the superior turbinate (Fig. 2). If the sphenoid ostia are not visible, however, the level of the superior turbinate can be used as a landmark for the level of the sphenoid sinus, which should correlate with a distance of approximately $1.5 \mathrm{~cm}$ above the choana. Both middle turbinates are lateralized, and this maneuver generally provides adequate working room. Care must be taken not to transmit force too close to the attachment of the middle turbinate to the lateral nasal wall because a fracture of the anterior cranial base and a CSF leak can occur.

Using a cottle elevator a mucosal incision is made on the posterior septum at the level of the sphenoid sinus ostium (Fig. 3). Then, using the soft-tissue shaver or through-biting instruments, a posterior septectomy is performed to remove approximately 1 to $1.5 \mathrm{~cm}$ of the posterior septum in front of the anterior face of the sphenoid sinus. We have found that the soft-tissue shaver provides a more rapid and precise removal of the septal mucosa than do the through-

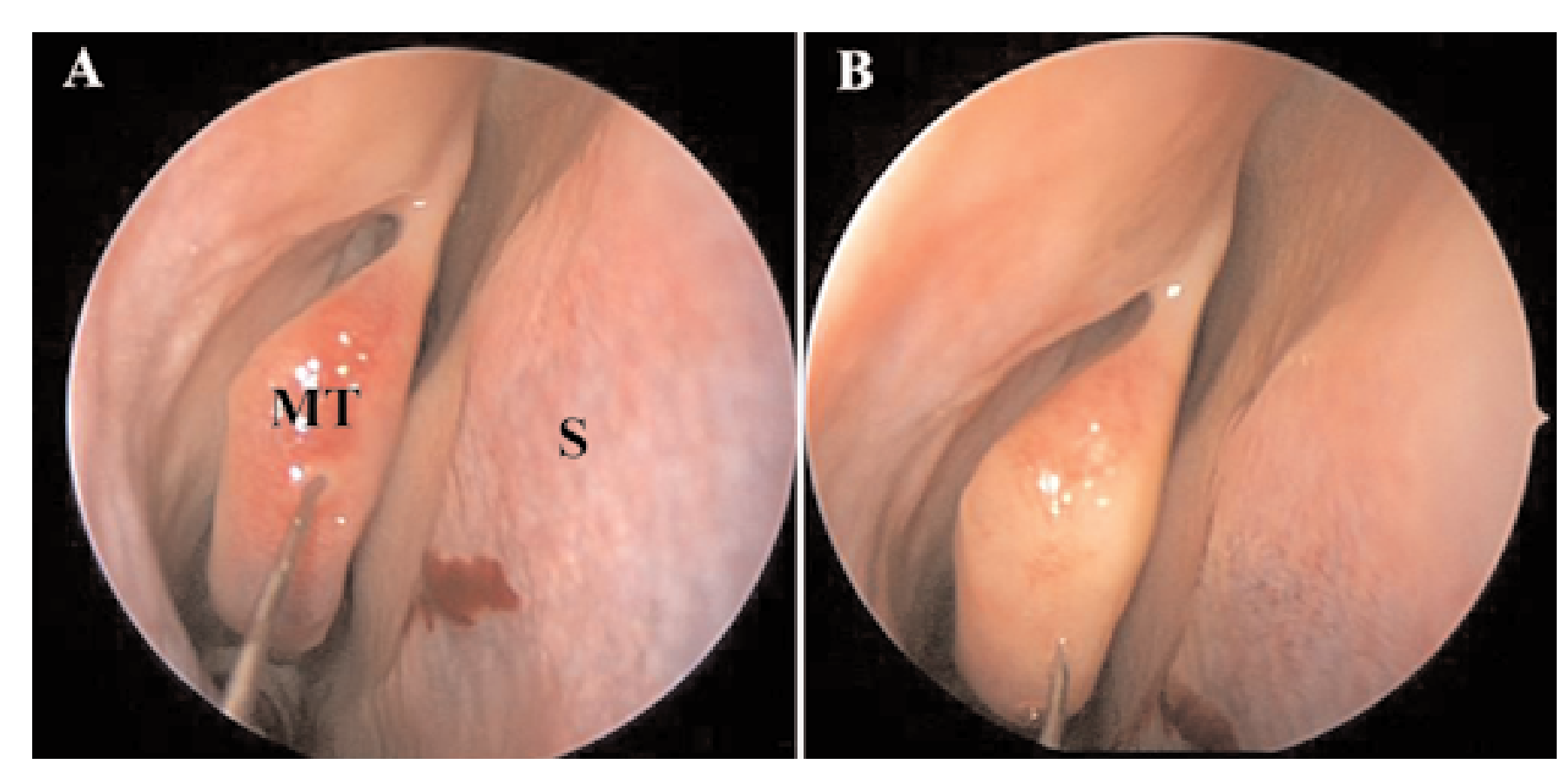

Fig. 1. Endoscopic views of mucosal injection within the right nostril just prior to (A) and after (B) injection of the middle turbinate (MT). S = nasal septum. 


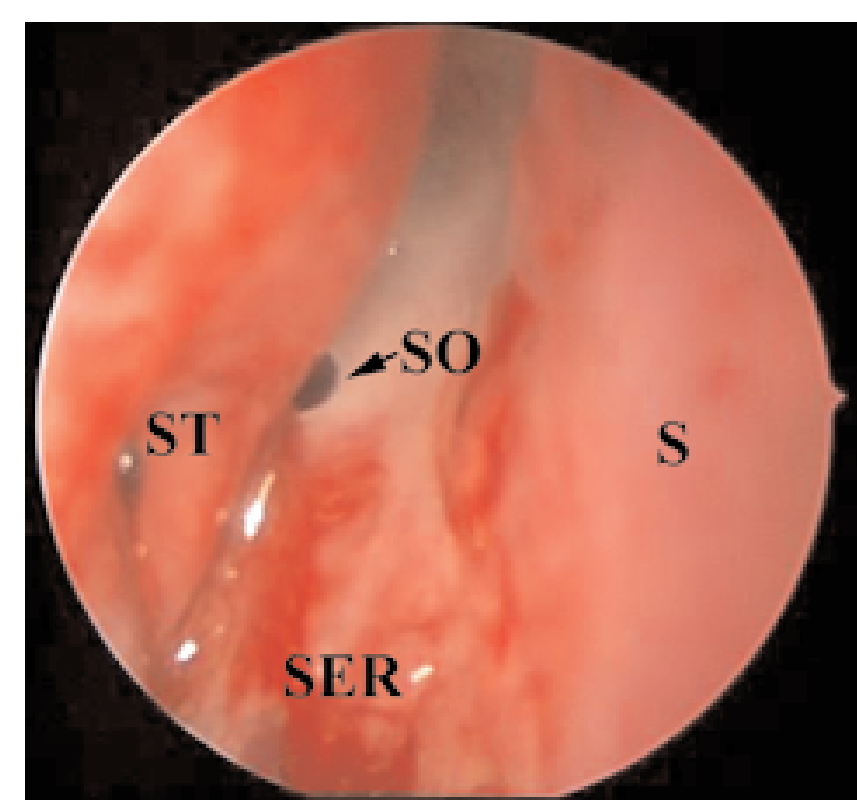

Fig. 2. Endoscopic view of the right sphenoid ostium (SO). The sphenoid ostium (arrow) can be found at the inferior third portion of the superior turbinate (ST) and provides an important landmark for the level of the sphenoid sinus. SER $=$ sphenoethomoid recess.

biting instruments. The posterior septectomy allows a binasal approach to the sella and prevents the septum from brushing against the end of the endoscope during the operation (Fig. 4). The mucosa over the sphenoid rostrum is elevated and removed again with either the soft-tissue shaver or through-biting instruments.

A wide anterior sphenoidotomy is performed including and lateral to both sphenoid ostia. The superior limit should allow visualization of the planum sphenoidale, the optic protuberances, and the opticocarotid recesses. During tumor resection, the endoscope will be positioned superolaterally; therefore, the sphenoidotomy will need to be large enough to accommodate the endoscope without obstructing the flow of instruments. The inferior limits of the sphenoidotomy must allow the suction to pass freely to the floor of the sella. In general, the vomer does not need to be completely removed to provide this exposure. Indeed, we advocate its preservation to provide an indicator of the anatomical midline. The sphenoidotomy may be performed using bone punches, the mucosal shaver, or a drill. During the inferolateral sphenoidotomy, care should be taken to avoid disrupting branches of the sphenopalatine artery because bleeding from these vessels can hinder the operation and cause postoperative epistaxis. Because this artery's position is regular, we have found the best way to avoid injury is to elevate the mucosa overlying the inferolateral rostrum separately from the bone. This step tends to elevate the vessel with the mucosa and allow bone removal without arterial injury.

Intraoperative C-arm fluoroscopy has not been necessary, except in exceptional situations, to determine the appropriate trajectory to the sella. Instead, the panoramic view of the sphenoid anatomy provides the information regarding the midline and limits of the sella. Under endoscopic guidance, however, the vomer is not the only mid-

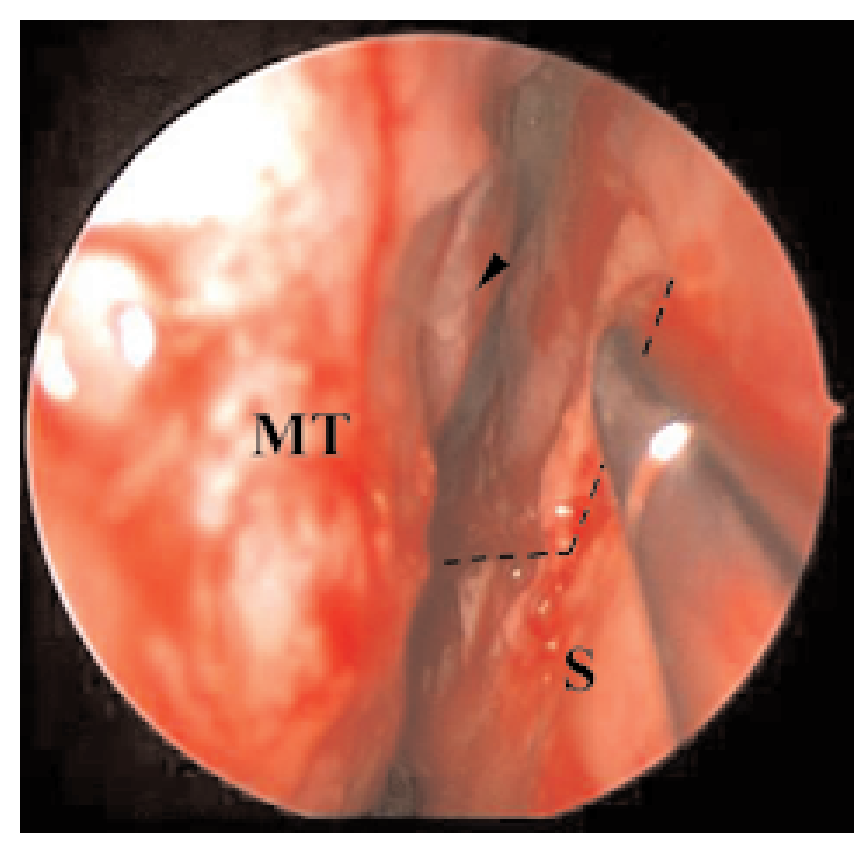

Fig. 3. Endoscopic view from within the right nasal cavity of a septal mucosal incision. After the sphenoid ostium is identified, the nasal septal mucosa is incised. This provides a cut surface to remove the septal mucosa and bone in order to accomplish a posterior septectomy. Arrowhead points to the superior turbinate, and the dotted line marks the mucosal incision.

line marker. The midpoint between the carotid protuberances or the opticocarotid recesses can also be used. Once the intersphenoid sinus septae are removed, the carotid and optic protuberances are identified, as are the opticocarotid recesses, clivus, sella, and planum (Fig. 5). We have found that all of these landmarks are not always visible. Nevertheless, enough of the anatomical landmarks are usually visible so that the sellar anatomy can be determined. If doubt continues, intraoperative video fluoroscopy is performed.

In general, the posterior septectomy and sphenoidotomy is performed using a two-handed mononostril technique, with the endoscope in one hand and an operative instrument in the other. The opposite nostril is accessed at times, particularly during the septectomy, but the majority of the endoscopy and dissection is performed via one nostril. From this point forward, the operation proceeds through a binasal three- or four-hand approach, with the endoscope and suction in one nostril and the operative instrument in the other. Interplay is required between the endoscopist and the surgeon. The endoscope should follow the instruments into and out of the nose and dynamically focus on the field of interest.

The sellar floor is then opened to the limits of the cavernous sinus laterally, the turberculum and planum opened superiorly, and the sellar floor opened inferiorly. We have not found it necessary to use a drill for the sellar opening, and we often simply fracture the sellar floor or open it with a fine chisel (Figs. 6 and 7). The dura mater is opened using a knife with a retractable blade designed by Cappabianca. The retractable blade has been useful in preventing mucosal injury when placing the knife within the nose. At times, a small vessel can be seen to exit the dura over the pituitary gland. We have found that this vessel correlates with the 


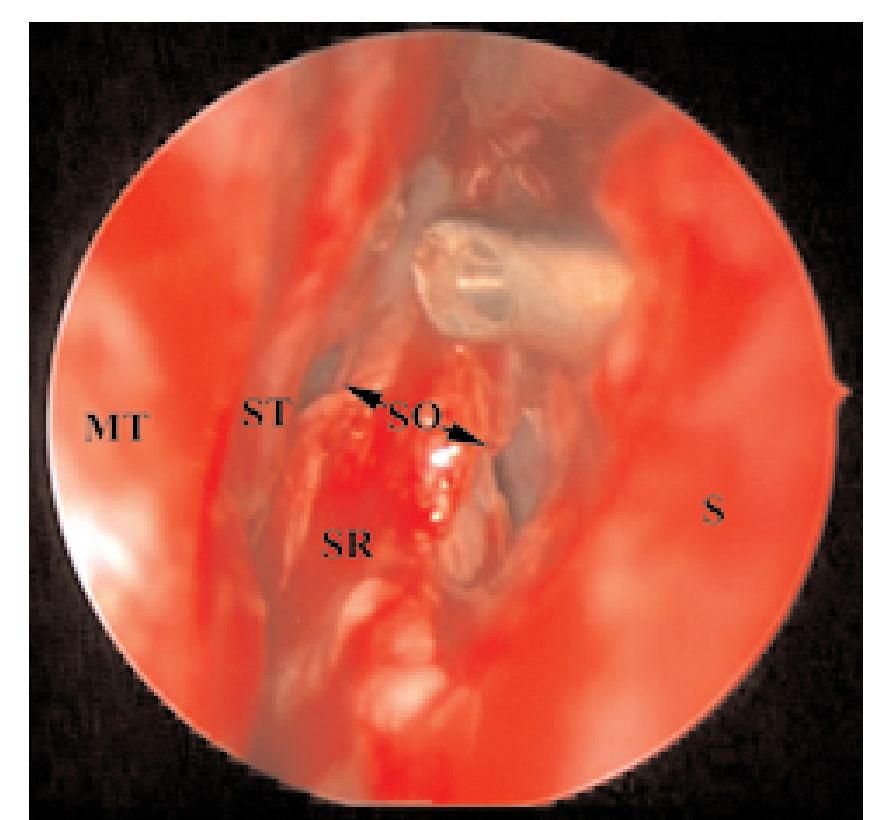

Fig. 4. Endoscopic view following the posterior septectomy but prior to the anterior sphenoidotomy. Both sphenoid ostia (arrows) are visible with the endoscope in the right nasal cavity. An instrument placed through the left nostril can be seen above both ostia. $\mathrm{SR}=$ sphenoid rostrum.

anatomical midline and can be used in addition to the other midline markers. The $0^{\circ}$ endoscope is used during the majority of the operation. Tumor resection proceeds in a manner similar to that of the microscopic adenomectomy, with a removal of the inferior, then the lateral, and finally the superior portions of the tumor against the diaphragm. The differences from the traditional microscopic approach are the more magnified views of areas that are out of a microscope's line of sight and the ability to move the endoscope in for dynamic views of areas of interest. With the 0 and $30^{\circ}$ endoscopes, the sellar floor, cavernous sinus walls, and diaphragm can be directly inspected (Fig. 8). During transdiaphragmatic dissection, the optic chiasm can be seen and chiasmal decompression (which is often the goal of surgery) can be confirmed instead of assumed. Using the microscope, the chiasm is rarely fully visualized. When the anatomy is not completely visualized, the $30^{\circ}$ endoscope can be used. Although this endoscope potentially provides better visualization, it tends to get more in the way of the superiorly placed operative instruments. We have thus found it more useful to place the $30^{\circ}$ endoscope inferiorly, with the suction and operative instruments above it.

Sellar reconstruction proceeds as we have previously described. ${ }^{13}$ If a CSF leak is noted during surgery, we place a fat graft within the sella. Appropriately sized fat grafts can be difficult to place within the nose, and at times a nasal speculum must be used to provide access. Often we will also place a dural substitute against the diaphragm and in a subdural plane over the fat. If no CSF leak is noted, we simply place Gelfoam within the sella. If adequate septal bone is not harvested during the procedure (as is the norm), a tailored bioabsorbable graft is sized using a 0.5 by 0.5 -inch pattie and placed in an extradural plane under the remaining sellar bone (Fig. 9). In the case of a CSF leak, we usually do not fill the sphenoid sinus with fat; if we do, however,

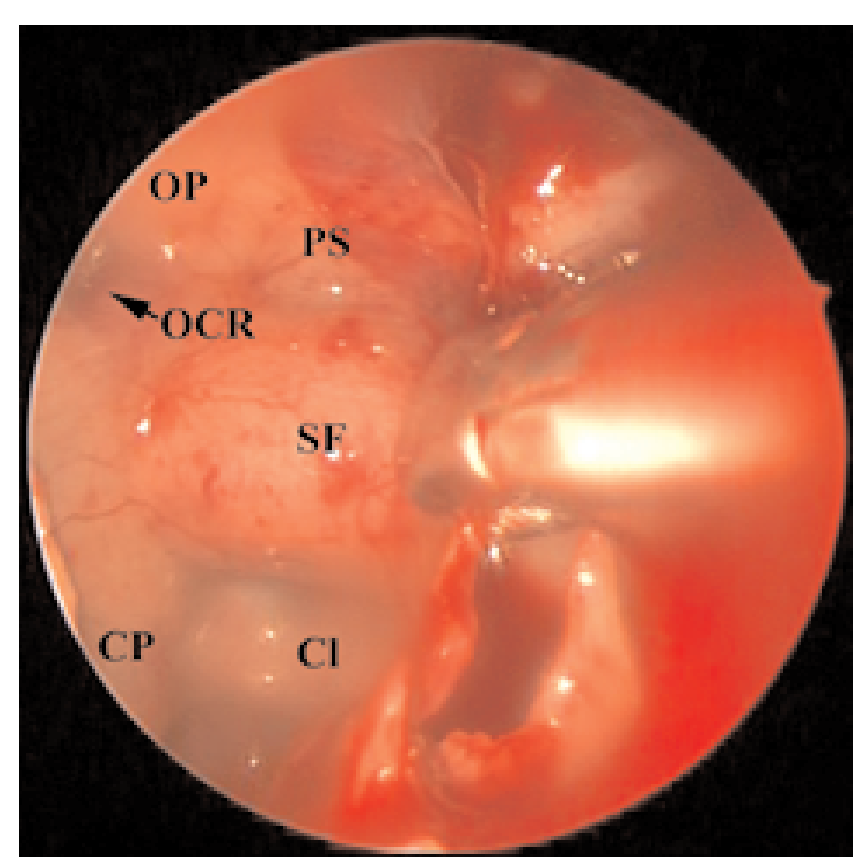

Fig. 5. Endoscopic view of the sphenoid sinus as the intersphenoid sinus septum is being removed. After the anterior sphenoidotomy is performed, the intersphenoid sinus septae are removed to expose the intrasphenoidal anatomy. Evident is the panoramic view of the sphenoid anatomy with the optic and carotid protuberances (OP and $\mathrm{CP}$, respectively), opticocarotid recess (OCR; arrow), clivus $(\mathrm{Cl})$, planum sphenoidale (PS), and sellar impression. $\mathrm{SF}=$ sellar floor.

we first remove the sphenoid mucosa. Otherwise, every attempt is made to preserve the sphenoid mucosa, and it is disrupted over the sella only.

At the end of the sellar reconstruction, the nose is irrigated and the nasal mucosa is inspected. The middle turbinates are medialized. Our bias is to "overmedialize" the turbinates. Although this step may lead to synechia between the middle turbinate and the septum, we believe that it prevents the more significant complication that can occur if the lateralized middle turbinate occludes the osteo-meatal complex. The nasopharynx, oropharynx, and stomach are then suctioned. Ordinarily, the nose is not packed. We use temporary nasal packing if a large CSF leak occurs and the sphenoid sinus has been packed with fat, particularly if we believe that the sphenoidotomy is too large to keep the fat in place. We maintain this packing overnight or until the second postoperative day. We do not routinely use lumbar drainage, a bladder catheter, or central lines. Postoperatively, patients receive oral decongestant agents for 2 weeks, saline nasal sprays hourly during waking hours, and oral antibiotic agents for 5 days. As with our microscopic adenomectomies, patients are not sent to the intensive care unit.

\section{DISCUSSION}

\section{Evolution of Surgical Technique}

Early in our experience we attempted to recapitulate the microscopic approach. The endoscope holder seemed to provide the closest approximation of the microscopic tech- 


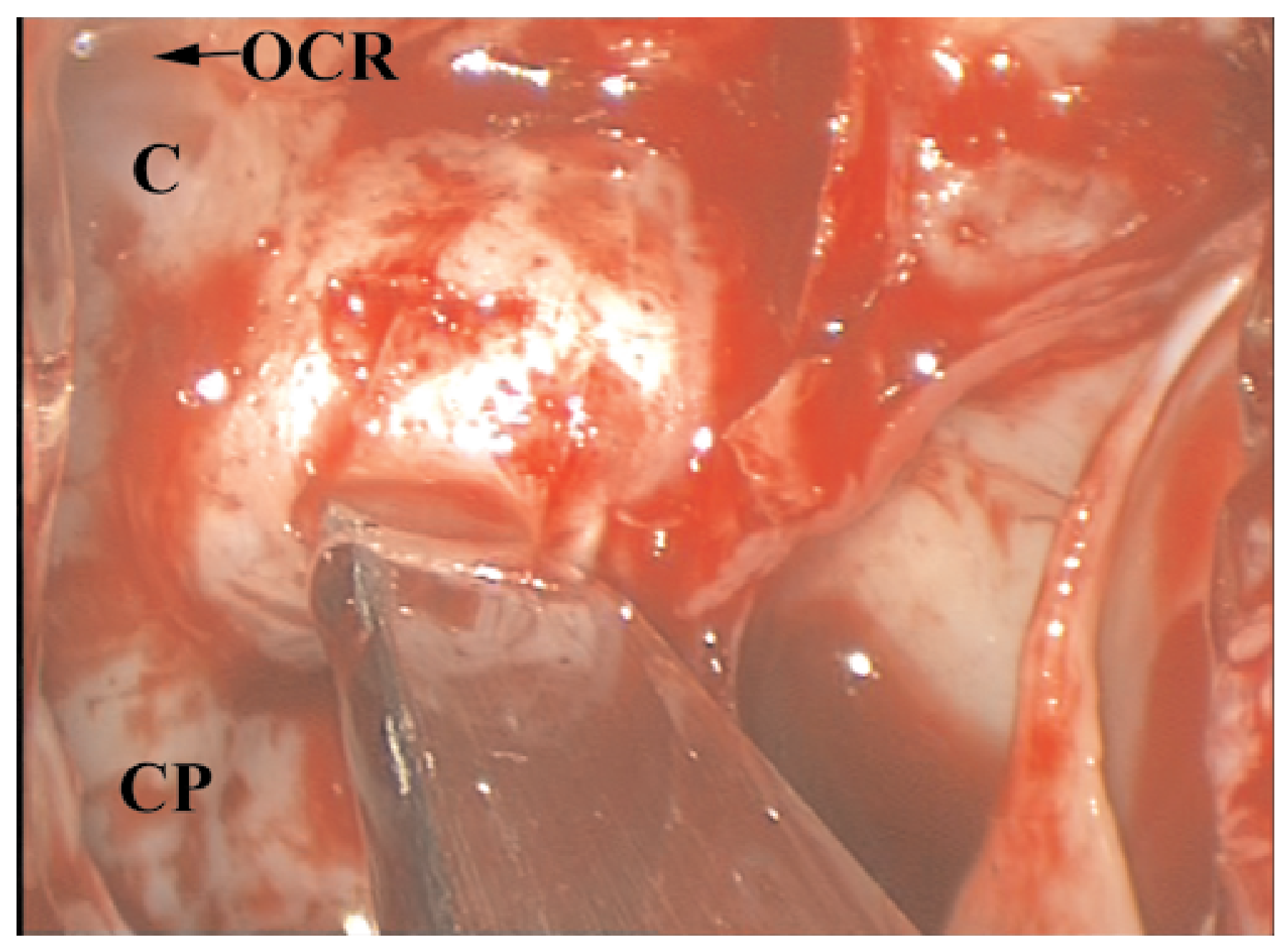

Fig. 6. Endoscopic view of the opening of the sellar floor. When the sellar floor is relatively thick, as is the case for many microadenomas, a chisel can be used to create a small opening in it. $\mathrm{C}=$ carotid artery.

nique. In our experience, however, the holder tends to negate the advantages of the endoscope. Current endoscopes have limited zoom capability; hence the surgery is generally performed with maximal zoom from the outset. The surgeon can obtain a pseudodepth perception from the dynamic movement of the endoscope relative to the operative instruments that, when fixed, is absent. Placing the endoscope in a fixed position likens the technique to a microscopic adenomectomy during which the surgeon has a wide-angle view but has limited depth perception and zoom views. Also, if the endoscope becomes occluded by blood beyond the cleaning ability of the irrigation system, it must be removed from the holder, cleaned, and then brought back into the field. This interruption tends to disrupt the flow of the operation significantly and, in situations of significant bleeding, can be disconcerting. Even with a wide anterior sphenoidotomy, the endoscope occasionally restricts the maneuverability of instruments and access to the tumor.

Using the three-hand technique as advocated by Kassam and others, ${ }^{16,17}$ the benefits of the endoscope can be realized. This maneuver requires cooperation between the endoscopist and the surgeon. The endoscopist can move in during the tumor resection, and focus closely on areas of in- terest. The pseudo-three-dimensional perspective is also greatly enhanced by the in-and-out movement of the endoscope. As instruments are moved in and out of the nose, the endoscope operator can follow the instruments, ensuring that they do not injure the nasal mucosa.

Over time we have experimented with the supine and semirecumbent positions. We prefer the latter but recognize certain limitations. The supine position increases the venous pressure and can cause significant bleeding from the cavernous sinus and tumor. As with the microscopic adenomectomy, significant bleeding can be the pivotal factor separating an inadequate tumor resection from a successful operation. Nevertheless, the supine position is more comfortable for the surgeon and causes less arm and shoulder fatigue. The arm strain associated with the semirecumbent position can be alleviated by standing on step stools, which allows the endoscopist to maintain elbows at his or her side. Because of the advantages of decreased venous pressure and more facile tumor removal, we advocate a semirecumbent position, with the back and head elevated approximately $20^{\circ}$.

The other keys to minimizing bleeding are adequate injection of vasoconstrictive agents, careful insertion and 


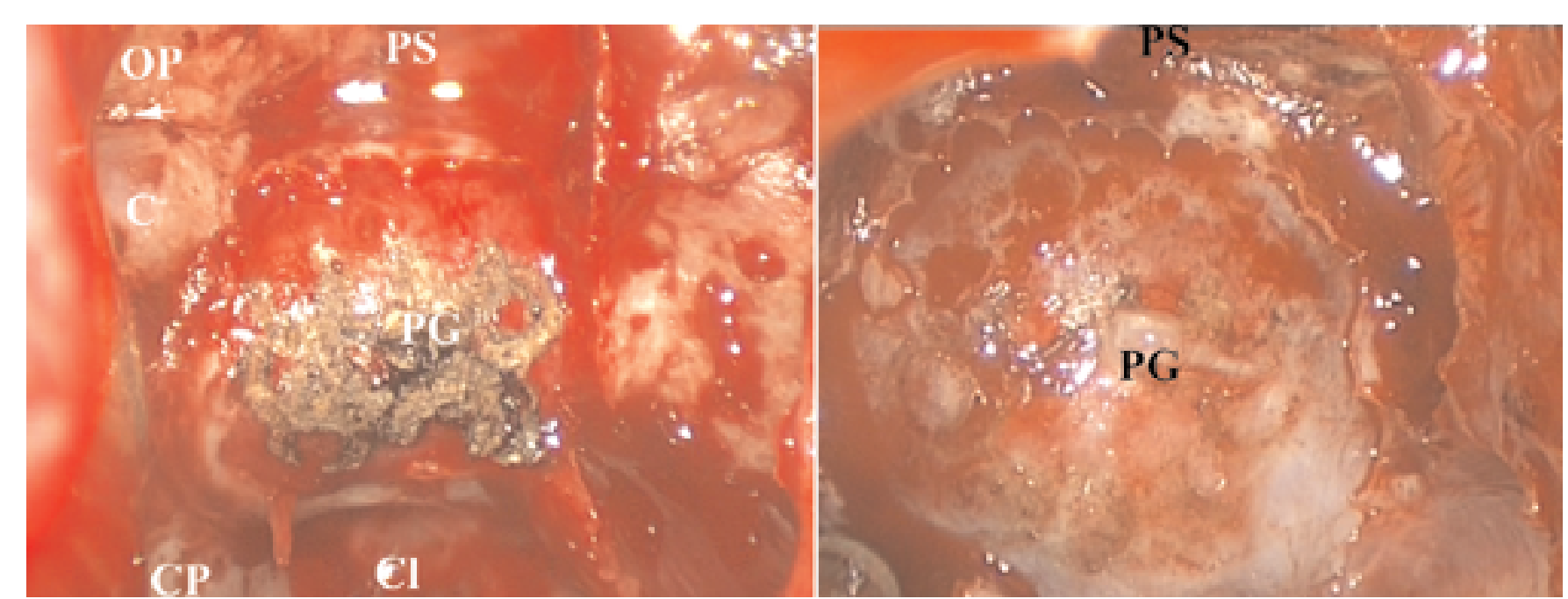

Fig. 7. Endoscopic views of the extent of sellar opening. The sellar floor is opened from cavernous sinus to cavernous sinus and from the planum sphenoidale to the clivus. A small ledge of bone is left intact bilaterally. This allows a graft to be placed at the end of the operation to reconstruct the sellar floor. Left: Example of a sellar opening prior to resection of an intrasellar microadenoma. Note the excellent view of the intrasphenoidal anatomy. Right: Example of the sellar opening prior to resection of a macroadenoma with suprasellar extension. Note the disruption of the normal sphenoidal anatomy. $\mathrm{PG}=$ pituitary gland.

removal of instruments, and use of deliberate and efficient maneuvers in performing the operation. Most sellar, cavernous sinus, and bone bleeding can be quickly controlled using FloSeal (Baxter Health Care Corp., Deerfield, IL). ${ }^{6}$ Its disadvantage is that it does not fully wash away with irrigation and can make inspecting for tumor remnants more difficult. An ideal bipolar instrument has not yet been introduced.

We also do not advocate routine partial middle turbinectomy because it is mostly unnecessary in the adult patient, except for those with an unusually small nose and nasal cavity. If it is performed routinely, the minimally invasive nature of the endoscopic procedure as compared with its microscopic transsphenoidal counterpart becomes dubious. Difficulties with crusting and delayed epistaxis become more prominent, and because nasal packing is more likely, patient discomfort significantly increases, again negating many of the advantages of the endoscopic approach. When middle turbinate lateralization does not suffice, partial middle turbinectomy along the inferior portion of the middle turbinate is performed.

\section{Advantages and Limitations of the Pure Endoscopic Adenomectomy}

To better understand the role of the pure endoscopic technique, it is helpful to understand the advantages and limitations of the microscopic adenomectomy. Among the important advantages of the microscope include its familiarity to the neurosurgeon. The operating microscope is standard equipment used for all types of neurosurgical procedures on the spine and brain. The microscope's benefit to the surgeon's comfort should not be underestimated. The microscopic view is three-dimensional, which is of enormous importance during tumor dissection, and it also has easily manipulated zoom and focus features. Once it is focused at maximum zoom, the surgeon can increase and decrease the zoom without needing to refocus. Furthermore, after the appropriate trajectory to the sella has been discerned, the operating microscope can easily maintain it, preventing unintentional breach of the anterior cranial fossa. Although a nasal speculum is used for a microscopic adenomectomy, it is not part of most pure endoscopic procedures. The speculum protects the nasal mucosa from injury by instruments during the operative procedure. Finally, the operating microscope is out of the surgical field and does not obstruct the entry or maneuvering of instruments into or out of the it. Because it is out of the surgical field, the microscopic view does not suffer from red out when bleeding fills the surgical field. For busy centers, the relative brevity of the microscopic adenomectomy allows for multiple cases to be addressed in a single day.

There are problems with the microscopic adenomectomy, however. Although the optics are excellent and changes in magnification can be performed easily, the field of view is narrow. This restriction necessarily means that some anatomical landmarks within the sphenoid sinus are not readily visualized. In our experience, it is quite rare to see the carotid or optic protuberances or the opticocarotid recesses microscopically. Although some may argue that because tumors are centrally located it is unnecessary to see these landmarks, their visualization does orient the surgeon to the anatomical midline and to the limits of the sella. Therefore, it is our standard practice to use C-arm videofluoroscopy or other forms of image guidance for all of our microscopic transsphenoidal operations. In addition, visualization with the microscopic alternative is limited by line of sight. The surgeon cannot see around corners, either toward the cavernous sinus or the suprasellar space. Visualization of the optic chiasm, confirming its decompression during transdiaphragmatic dissection, is rarely possible microscopically. The standard sublabial or transseptal approaches can also be 


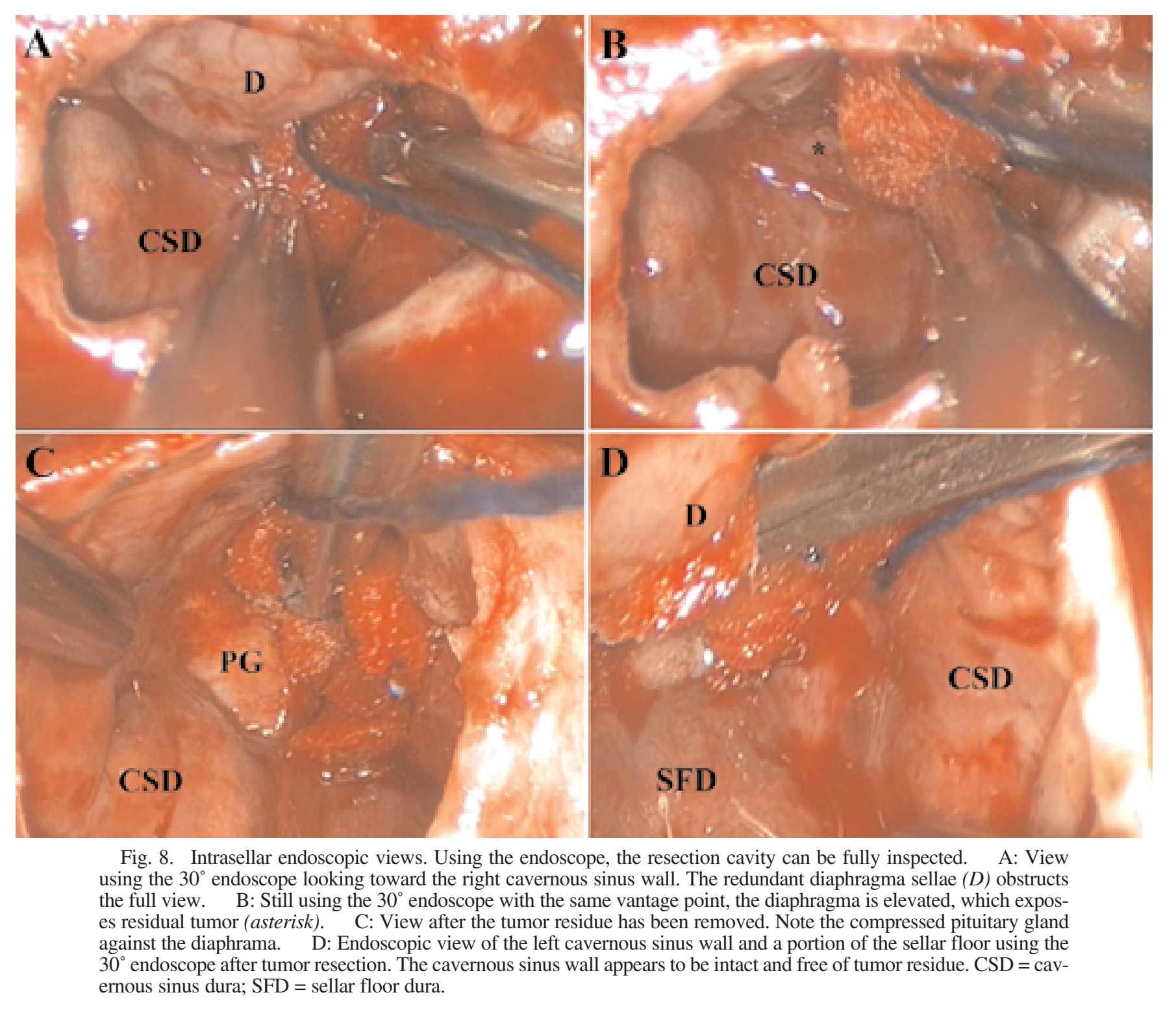

associated with sinonasal complications and with significant patient discomfort early on in the perioperative period. Nasal packing causes facial pain and headache in as many as $35 \%$ of patients, and the procedures can cause rhinological complaints including alveolar numbness, anosmia, saddle nose deformity, and nasal septal perforations. ${ }^{20-22}$ These complications are less pronounced with the endonasal transseptal approach, but they do continue to occur. The more minimally invasive approaches of the septal pushover and direct sphenoidotomy do not require nasal packing and are associated with fewer sinonasal complications. ${ }^{13,25}$ Nevertheless, these benefits come at the cost of a more limited exposure, an off-midline trajectory tending to lead to the carotid, and a confined working area.

The endoscopic approach also presents difficulties. Although many neurosurgeons perform ventriculoscopy, nasal endoscopy is much less familiar. This means that most neurosurgeons, who typically perform their own microscopic exposures, will need to find an interested ENT sur- geon. The three-hand technique requires that a facile endoscopist must stay throughout the entire operation. Typically during microscopic approaches, the ENT surgeon performs the transsphenoidal exposure and nasal closure but is not necessarily present for the tumor resection. When neurosurgeons perform these neuroscopic operations without ENT surgeons, the operative times during the learning curve are significantly longer than with microscopic adenomectomy. Whereas the latter can be easily taught to junior neurosurgery residents, teaching them both the surgical anatomy and the use of the endoscope can be an imposing task. Other major limitations include the zoom and focus capacity of the endoscope. Because the endoscope has a relatively limited zoom capability, our tendency is to use it at maximum zoom at all times. To obtain magnification, the endoscope is brought closer to the region of interest, requiring frequent refocus maneuvers that are not as easily performed as with the microscope.

Before the endoscopic surgeon fully understands the 


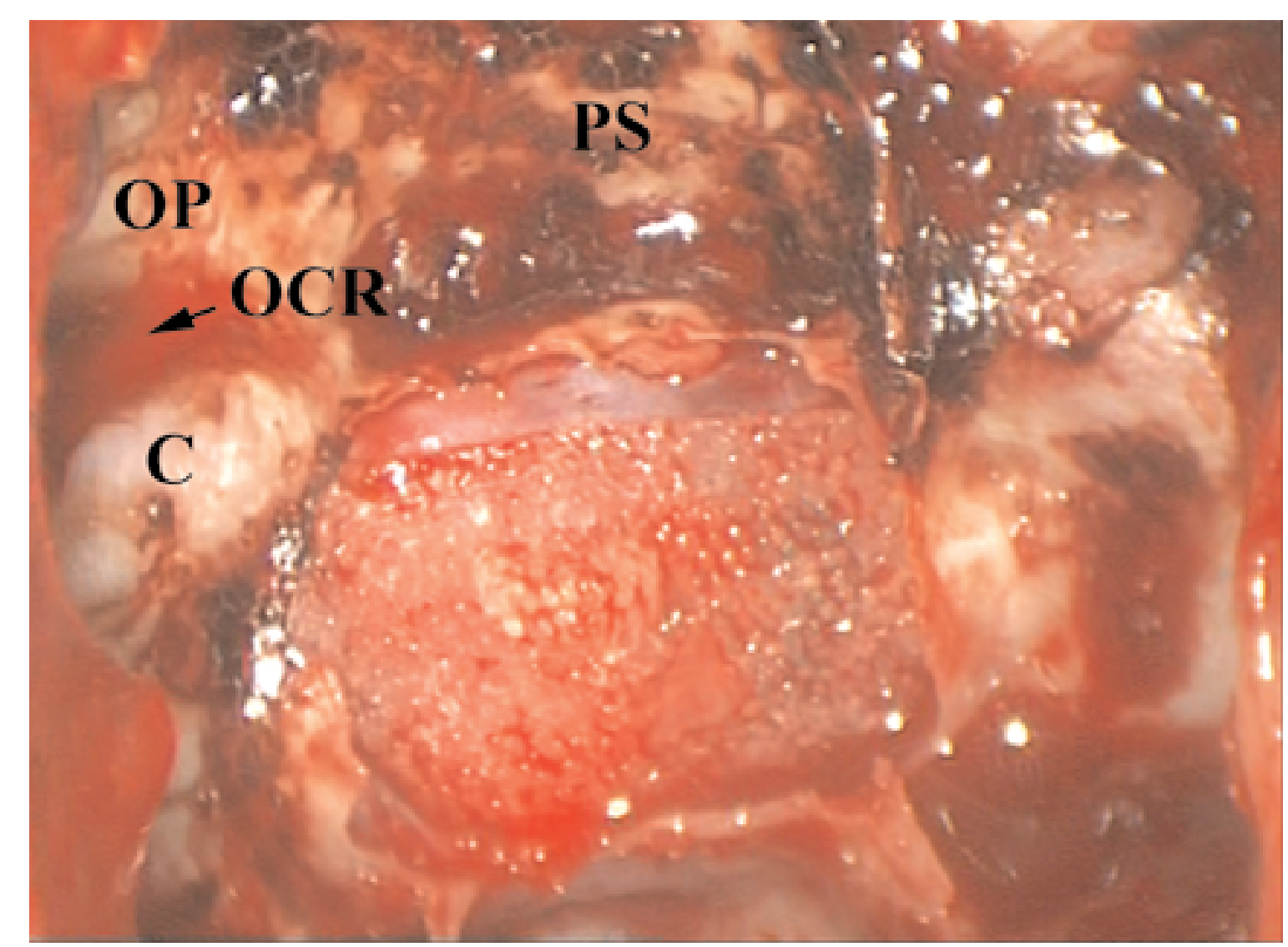

Fig. 9. Endoscopic view of sellar floor reconstruction. After tumor resection, the sellar floor is reconstructed by placing a tailored bioabsorbable graft in the extradural plane under the remaining edge of sellar bone.

depth perception provided by the dynamic interaction between surgical instruments and the endoscope, it is possible to dissect into the posterior pituitary, cavernous sinus, and diaphragm overaggressively and cause more complications, such as CSF leaks and diabetes insipidus. Because instruments enter the field out of the line of sight of the endoscope and because no nasal speculum is used, the nasal mucosa along the septum and the medial wall of the middle turbinate can be injured in the course of the operation. This damage can be minimized by lateralizing the middle turbinates and by careful entry of surgical instruments into the nose. Also, we believe it is important to overmedialize the middle turbinates at the end of the operation to avoid maxillary sinus obstruction. During the endoscopic sphenoidotomy, the posterior septal branches of the sphenopalatine artery are also at greater risk than during transseptal microscopic approaches. In these approaches, the mucosa containing this vessel are elevated from the sphenoid rostrum and are thereby protected. The direct sphenoidotomy places these vessels at greater risk of damage and of early postoperative epistaxis.

It is perhaps an overstatement to assert that the endoscope is less invasive than the microscope. The endoscopic approach may seem so to the patient initially because there is no nasal packing, but the endoscopic approach, as we assert, disturbs and removes more anatomical structures than the various standard microscopic approaches (sublabi- al, endonasal transseptal, and direct sphenoidotomy). The sphenoidotomy is larger so that the endoscope does not impede the necessary maneuvering of the operative instruments. To achieve the same maneuverability of the operative instruments as in the microscopic adenomectomy, a binasal technique is used that requires a posterior partial septectomy. Although not routine, a partial middle turbinectomy is also performed at times, an action never necessary when performing a microscopic adenomectomy. All of these maneuvers are undertaken in an effort to provide an exposure that allows the surgeon to use two instruments and the endoscope.

Indeed, it can be reasonably argued that these factors necessarily mean that the binasal endoscopic technique is more invasive than the microscopic adenomectomy. The mononostril approaches as advocated by Jho and Carrau ${ }^{15}$ and Cappabianca and colleagues, ${ }^{2,3}$ however, can reasonably be considered less invasive. We simply have not been able to achieve the same operative maneuverability in most cases using a mononostril approach. Nevertheless, each of the microscopic approaches requires reflection of some portion of the nasal septum, an act that can lead to nasal septum deviation. The microscopic sublabial and transseptal approaches also require anterior septum dissection, creating the risks of nasal septum perforation, septal hematoma, alveolar numbness, and saddle nose deformity. ${ }^{20-22,25}$ All of these risks are relatively low, and the choice to use the en- 
doscopic in lieu of the microscopic technique should not be in the pursuit of a less invasive approach, but because of the advantages provided by the endoscopic procedure.

Because the technique is relatively new, appropriate instruments are still in development. Still needed are a proper bipolar instrument and slotted suckers with angled tips to work in the regions visualized by the endoscope but inaccessible with direct suction. Because current angled suction devices are not slotted, problems can arise when placing the suction into the cavernous sinus or suprasellar region. Smaller endoscopes with more zoom range and focus capacity also need to be developed. To foster a wider application of this technique, a standardized set of instruments required to begin performing these operations must be developed.

The endoscope does afford significant advantages both during the surgical approach and during tumor resection. It provides panoramic views of areas outside of a microscope's line of sight and allows dynamic, magnified inspection of areas of interest. During the approach, the wide views of the nasal anatomy allow the surgeon to discern the appropriate level of entry into the sphenoid sinus more easily (either by visualizing the sphenoid ostia or the level of the superior turbinates). This wide-angle view and superior visualization of the sphenoid anatomy provides excellent information regarding superior and inferior limits of the sella and the anatomical midline. These factors obviate the use of intraoperative videofluoroscopy in all but the most exceptional cases. Visualization of the carotid protuberances is rarely possible with the operating microscope, and confirmation of their precise location does at least improve surgeon comfort.

During tumor resection, particularly with microadenomas and sellar macroadenomas, exceptionally magnified views of the tumor/gland interface can be discerned and dissection enabled. Even with the $0^{\circ}$ endoscope, the sellar floor, cavernous sinus walls, and diaphragm can be directly inspected. The exception is the proximal portion of the cavernous sinus wall just posterior to the dural opening on the ipsilateral side to the endoscope. This portion of the cavernous sinus wall is more difficult to see, but it can be done by placing the endoscope within the other nostril (the "cross-court" approach). During transdiaphragmatic dissection, the optic chiasm can be seen and chiasmal decompression, which is often the goal of surgery, can be confirmed. Using the microscope, the sellar floor, cavernous sinus walls, and chiasm are rarely fully visible. When the anatomy is not completely apparent, the $30^{\circ}$ endoscope can be used. This particular endoscope does tend to conflict with superiorly placed operative instruments. We have found it more useful to place the $30^{\circ}$ endoscope inferiorly, with the suction and operative instruments above it, or to place it in one nostril and introduce the operative instruments through the other. It is not yet known whether these advantages will result in higher remission rates for secretory adenomas and lower recurrence rates for nonfunctioning adenomas. The early complication rate does not appear to be increased, but longer follow-up periods are needed so that conclusions regarding efficacy can be drawn. ${ }^{3}$

The endoscopic adenomectomy rarely requires nasal packing unless there is a significant CSF leak. As has been shown by Zada and colleagues, ${ }^{25}$ foregoing nasal packing significantly decreases patient discomfort and affects pa- tient satisfaction. Although we have not yet begun to assess this aspect systematically, our impression is that patients undergoing endoscopic surgery experience much less postoperative discomfort.

The endoscopic adenomectomy can be associated with alterations in the sense of smell, but it is not associated with the complications of alveolar numbness, nasal septal perforations and deviations, and saddle nose deformities that are common with the traditional transsphenoidal sublabial or transseptal microsurgical approaches. The endoscopic exposure is most like the direct sphenoidotomy as described by Griffith and Veerapen, ${ }^{8}$ and now advocated by Zada, et al. ${ }^{25}$ The main anatomical difference is that the three- or four-hand binasal endoscopic approach requires a partial posterior septectomy. During the endoscopic approach, the nasal mucosa is at greater risk of injury because no nasal speculum is used. With the microscopic direct sphenoidotomy, the posterior septum is simply reflected laterally. Zada and colleagues ${ }^{25}$ have reported that one fifth of patients undergoing the direct sphenoidotomy required a relaxing alar incision; such incisions are not necessary during endoscopic cases. In our view, the alar incision is a major drawback of the microscopic direct sphenoidotomy. The most significant deficiency of the direct microscopic adenomectomy, however, is the narrow exposure and slightly offmidline trajectory. Cadaveric or clinical studies directly comparing these approaches would be helpful.

\section{CONCLUSIONS}

The pure endoscopic adenomectomy is a safe, effective approach to sellar region tumors that offers several advantages over the microscopic approach. It provides an excellent wide-angle and magnified view of the operative anatomy, and although it requires more anatomical exposure it remains within the group of minimally invasive approaches to the sella. New instruments and smaller endoscopes with more focus and zoom capacity still need to be developed. Unbiased understanding of the endoscopic limitations and advantages will better allow the pure endoscopic adenomectomy to realize its place in transsphenoidal surgery. Direct prospective comparison of outcomes at a single institution would provide important insights.

\section{Disclaimer}

The authors have no financial interest in any of the instruments or devices discussed in this article.

\section{References}

1. Apuzzo MLJ, Heifetz MD, Weiss MH, Kurze T, et al: Neurosurgical endoscopy using the side-viewing telescope. Technical note. J Neurosurg 46:398-401, 1977

2. Cappabianca P, Cavallo LM, Colao AM, et al: Surgical complications associated with the endoscopic endonasal transsphenoidal approach for pituitary adenomas. J Neurosurg 97:293-298, 2002

3. Cappabianca P, de Divitiis E: Endoscopy and transsphenoidal surgery. Neurosurgery 54:1043-1050, 2004

4. Cushing H: Partial hypophysectomy for acromegaly. With remarks on the function of the hypophysis. Ann Surg 50: 1002-1017, 1909

5. Elias WJ, Chadduck JB, Alden TD, et al: Frameless stereotaxy for transsphenoidal surgery. Neurosurgery 45:271-275, 1999 
6. Ellegala DB, Maartens NF, Laws ER Jr: Use of FloSeal hemostatic sealant in transsphenoidal pituitary surgery: technical note. Neurosurgery 51:513-515, 2002

7. Frank G, Pasquini E, Calbucci F: Endoscopic approach to the cavernous sinus via an ethmoido-pterygo-sphenoidal route, in 5th European Skull Base Society Congress: Copenhagen, June 15-17, 2001: Additional Abstracts. Copenhagen: ESBSC, 2001, p 009b (Abstract)

8. Griffith HB, Veerapen R: A direct transnasal approach to the sphenoid sinus. Technical note. J Neurosurg 66:140-142, 1987

9. Guiot G: Transsphenoidal approach in surgical treatment of pituitary adenomas: general principles and indications in nonfunctioning adenomas, in Kohler PO, Ross GT (eds): Diagnosis and Treatment of Pituitary Tumors. Amsterdam: Excerpta Medica, 1973, pp 159-178

10. Halstead AE: Remarks on the operative treatment of tumors of the hypophysis. With the report of two cases operated on by an oronasal method. Trans Am Surg Assoc 28:73-93, 1910

11. Hardy J: Transsphenoidal microsurgery of the normal and pathological pituitary. Clin Neurosurg 16:185-217, 1969

12. Hirsch O: Endonasal method of removal of hypophyseal tumors. With a report of two successful cases. JAMA 5:772-774, 1910

13. Jane JA Jr, Thapar K, Alden TD, et al: Fluoroscopic frameless stereotaxy for transsphenoidal surgery. Neurosurgery 48: 1302-1307, 2001

14. Jane JA Jr, Thapar K, Kaptain GJ, et al: Pituitary surgery: transsphenoidal approach. Neurosurgery 51:435-442, 2002

15. Jho HD, Carrau RL: Endoscopic endonasal transsphenoidal surgery: experience with 50 patients. J Neurosurg 87:44-51, 1997

16. Kassam A, Snyderman CH, Mintz A, et al: Expanded endonasal approach: the rostrocaudal axis. Part I. Crista galli to the sella turcica. Neurosurg Focus 19(1):E3, 2005

17. Kassam A, Snyderman CH, Mintz A, et al: Expanded endonasal approach: the rostrocaudal axis. Part II. Posterior clinoids to the foramen magnum. Neurosurg Focus 19(1):E4, 2005

18. Kocher T: Ein Fall von Hypophysis-Tumor mit operativer Heilung. Dtsch Z Chir 100:13-37, 1909

19. Schloffer H: Erfolgreiche Operationen eines Hypophysentumors auf Nasalem Wege. Wien Klin Wochenschr 20:621-624, 1907

20. Sharma K, Tyagi I, Banerjee D, et al: Rhinological complications of sublabial transseptal transsphenoidal surgery for sellar and suprasellar lesions: prevention and management. Neurosurg Rev 19:163-167, 1996

21. Sherwen PJ, Patterson WJ, Griesdale DE: Transseptal, transsphenoidal surgery: a subjective and objective analysis of results. J Otolaryngol 15:155-160, 1986

22. Spencer WR, Levine JM, Couldwell WT, et al: Approaches to the sellar and parasellar region: a retrospective comparison of the endonasal-transsphenoidal and sublabial-transsphenoidal approaches. Otolaryngol Head Neck Surg 122:367-369, 2000

23. von Eiselsberg A: The operative cure of acromegaly by removal of a hypophysial tumor. Ann Surg 48:781-783, 1908

24. Wilson WR, Laws ER Jr: Transnasal septal displacement approach for secondary transsphenoidal pituitary surgery. Laryngoscope 102:951-953, 1992

25. Zada G, Kelly DF, Cohan P, et al: Endonasal transsphenoidal approach for pituitary adenomas and other sellar lesions: an assessment of efficacy, safety, and patient impressions. J Neurosurg 98:350-358, 2003

Manuscript received October 15, 2005.

Accepted in final form December 1, 2005.

Address reprint requests to: John A. Jane Jr., M.D., Department of Neurological Surgery, Box 800212, University of Virginia Health System, Charlottesville, Virginia 22908. email: johnjanejr@ virginia.edu 